\title{
Doppler- und Duplexsonografie in der Diagnostik des irreversiblen Hirnfunktionsausfalls
}

\section{Doppler and Duplex Sonography for the Diagnosis of Irreversible Loss of Brain Function}

\author{
Autoren \\ M. Kaps ${ }^{1}$, S. Schreiber ${ }^{2}$, U. Walter \\ Institute \\ ${ }^{1}$ Klinik für Neurologie am Standort Gießen, Universitätsklinikum Gießen und Marburg \\ ${ }^{2}$ Klinik für Neurologie, Campus Mitte, Charité - Universitätsmedizin Berlin \\ ${ }^{3}$ Klinik und Poliklinik für Neurologie, Universitätsmedizin Rostock
}

\section{Schlüsselwörter \\ - Hirntod \\ - Zusatzverfahren \\ - Dopplersonografie \\ - Duplexsonografie \\ - zerebraler Zirkulationsstill- stand}

\section{Key words}

- brain death

- ancillary tests

- doppler sonography

- duplex sonography

- cessation of cerebral blood circulation

\section{Bibliografie}

DOI http://dx.doi.org/

10.1055/s-0041-111531

Klin Neurophysiol 2016;

47: 26-32

(c) Georg Thieme Verlag KG

Stuttgart · New York

ISSN 1434-0275

Korrespondenzadresse

Prof. Dr. med. Manfred Kaps

Neurologische Klinik

Universitätsklinikum Giessen

und Marburg

Klinikstraße 33

35392 Gießen

Manfred.Kaps@neuro.med.

uni-giessen.de

\section{Zusammenfassung}

$\nabla$

Extra- und transkranielle Ultraschallverfahren sind weltweit als apparative Zusatzdiagnostik zur Feststellung des zerebralen Zirkulationsstillstandes, wie er für den nicht behebbaren Ausfall der Hirnfunktion charakteristisch ist, anerkannt. In die aktuelle Vierte Fortschreibung der Richtlinie der Bundesärztekammer zur Feststellung des irreversiblen Hirnfunktionsausfalls („Hirntod“) haben neuerdings auch die Duplexsonografie und die CT-Angiografie Eingang gefunden. Deutlich ausführlicher und präziser werden formale und praktische Ausführungsbestimmungen formuliert, und vor allem werden an die Qualifikation der Untersucher höhere Anforderungen gestellt. Dieser Artikel gibt eine Einführung in die jetzt geltende Richtlinie der Bundesärztekammer und kommentiert deren Inhalte, soweit sonografische Verfahren als ergänzende Diagnostik angesprochen sind.

\section{Einleitung}

$\nabla$

Eine der wesentlichen Neuerungen der aktuellen vierten Fortschreibung der Richtlinie der Bundesärztekammer zur Feststellung des irreversiblen Hirnfunktionsausfalls („Hirntod“) - im folgenden Text mit RL4-BÄK bezeichnet - ist die Aufnahme der Duplexsonografie und der computertomografischen Angiografie (CTA) als Nachweisverfahren für den zerebralen Zirkulationsstillstand [1]. Außerdem wurden in der RL4-BÄK umfangreiche Präzisierungen vorgenommen, die in den separat angefügten „Begründungen“ insbesondere auch für die Neurosonologie noch weiter erläutert sind.

Weltweit und unabhängig von der geografischen Region ist die transkranielle Dopplersonografie (TCD) nach dem EEG die am häufigsten angewendete Zusatzuntersuchung („ancillary test“); in 5\%

\section{Abstract \\ $\nabla$}

Extra- and transcranial ultrasound is accepted worldwide in brain death protocols as a valid "ancillary" method to prove brain circulatory arrest. Duplex sonography and CT angiography were newly incorporated in the fourth update of the German Medical Association's guideline for the determination of the irreversible loss of brain function ("brain death") along with formal and practical specifications regarding the diagnostic procedure. The guideline also addresses the qualification required of the investigators. This article gives an introduction to the updated regulations of the German Medical Association and comments upon the section pertaining to neurovascular ultrasound. der Länder ist die TCD sogar verbindlich vorgeschrieben, während sie in den USA nur optional eingesetzt wird [2]. In Deutschland und auch international ist die TCD das am häufigsten eingesetzte Verfahren zur Feststellung des zerebralen Zirkulationsstillstandes, je nach länderspezifischen Richtlinien vor der CTA und der selektiven arteriellen Katheter-Angiografie [2-8]. Die verschiedenen nationalen Empfehlungen weisen dabei große Übereinstimmung auf in Hinblick auf technische Parameter und die pathognomonischen sonografischen Befunde des Zirkulationsstillstandes (systolische Spitzen, biphasische Strömungssignale), unterscheiden sich aber teilweise in der Festlegung der Anzahl der minimal zu untersuchenden Gefäße. Bspw. wird in Lateinamerika die TCD-Untersuchung nur der beiderseitigen A. cerebri media und der A. basilaris gefordert [7], in Übereinstimmung mit der Empfeh- 
lung einer nordamerikanischen Expertengruppe [8], während in Mitteleuropa und Japan die Untersuchung der beiderseitigen A. cerebri media, der beiderseitigen $A$. carotis interna sowie der beiderseitigen $A$. vertebralis gefordert wird $[1,9]$. Übereinstimmend mit den deutschen Richtlinien wird die Bedeutung der TCD hervorgehoben in Situationen, in denen der Apnoe-Test oder die Untersuchung einzelner Hirnstammreflexe nicht durchgeführt werden kann oder trotz Einhaltung strenger Regeln Unsicherheit bezüglich der Wirkung zentral dämpfender Medikamente auf die klinischen Symptome des Hirnfunktionsausfalls besteht $[1,2,4,7]$. Die Neuaufnahme der transkraniellen farbkodierten Duplexsonografie (TCCS) als Verfahren zum Nachweis des zerebralen Zirkulationsstillstandes ist den lateinamerikanischen und deutschen Richtlinien gemeinsam [1,7]. Die folgenden Ausführungen nehmen Bezug auf den zitatweise kursiv gedruckten Originaltext der RL4-BÄK sowie die Empfehlungen der Deutschen Gesellschaft für Klinische Neurophysiologie und Funktionelle Bildgebung (DGKN) zur Diagnostik des irreversiblen Hirnfunktionsausfalls $[1,10]$. Um dem Leser einen schnellen Überblick zu gewähren, werden neue Inhalte der RL4-BÄK im Text besonders hervorgehoben und punktuell kommentiert.

\section{Einsatz der Doppler-|Duplexsonografie \\ $\nabla$}

Die Doppler- bzw. Duplexsonografie kann bei allen Hirnschädigungen und in jedem Lebensalter zum Nachweis des zerebralen Zirkulationsstillstandes eingesetzt werden [9-17]. Beim Nachweis des zerebralen Zirkulationsstillstandes sind potentiell reversible Ursachen der klinischen Symptome des Hirnfunktionsausfalls ausgeschlossen und es kann die Irreversibilität des Hirnfunktionsausfalls bei Erwachsenen und Kindern ab dem vollendeten 2. Lebensjahr ohne Wartezeit und klinische Verlaufsuntersuchungen festgestellt werden [1]. Bei Kindern in einem Alter unterhalb des vollendeten 2. Lebensjahres kann die Doppler-/Duplexsonografie ebenfalls zum Irreversibilitätsnachweis eingesetzt werden, muss dann aber nach jeder der beiden klinischen Untersuchungen durchgeführt werden [1]. Voraussetzung für den Einsatz dieser Methode ist ein altersentsprechend suffizienter arterieller Mitteldruck, der bei Erwachsenen mehr als $60 \mathrm{mmHg}$ betragen muss [1]. Bei Kindern muss ebenfalls ein suffizienter arterieller Mitteldruck vorliegen, der jedoch in Abhängigkeit von Alter und Körpergröße kleinere Werte annehmen kann ( $\bullet$ Tab. 1$)[18,19]$.

\section{Pathophysiologie und methodische Limitationen \\ $\nabla$}

Die charakteristischen doppler-/duplexsonografischen Signale eines zerebralen Zirkulationsstillstandes sind Folge eines pathologischen Anstiegs des intrakraniellen Drucks über den arteriellen Mitteldruck ( $\bullet$ Abb. 1). Sorgfältiges Monitoring von beatmeten Intensivpatienten mit schweren Hirnschädigungen beinhaltet ohnehin regelmäßige transkranielle Doppler- oder farbduplexsonografische Untersuchungen, sodass verfolgt werden kann, ob und wie sich der typische Befund des irreversiblen zerebralen Kreislaufstillstandes allmählich entwickelt. Speziell hierin liegt ein besonderer diagnostischer Wert der Sonografie. Es wurde zudem eine Zeitabhängigkeit der Entwicklung der charakteristischen doppler-/duplexsonografischen Signale auch nach Feststellung der klinischen Symptome des Hirnfunktions-
Tab. 1 Altersabhängige Mindestwerte des arteriellen Mitteldrucks für die Durchführung der Doppler-/Duplexsonografie zum Nachweis des zerebralen Zirkulationsstillstandes.

\begin{tabular}{|ll} 
Alter [Jahre] & Mindestwert des arteriellen Mitteldruckes [mm Hg] \\
1 & 35 \\
2 & 40 \\
\hline $3-4$ & 45 \\
$5-7$ & 50 \\
$8-10$ & 55 \\
$>10$ & 60
\end{tabular}

Diese Werte entsprechen näherungsweise den publizierten $5 \%$-Perzentilen des arteriellen Mitteldruckes bei Kindern mit mittlerer Körpergröße der jeweiligen Altersgruppe (jeweils mittlerer der geschlechtsspezifisch angegeben Werte des arteriellen Mitteldruckes) [18]. Wenn die Körpergröße eines Kindes deutlich von seiner Altersnorm abweicht, sollte der individuelle Richtwert einer differenzierteren Tabelle entnommen werden [18]

ausfalls beschrieben, was einen potenziellen Einfluss auf die Sensitivität der Sonografie hat. So wurde bis zu $6 \mathrm{~h}$ nach der klinischen Diagnose der zerebrale Zirkulationsstillstand mittels TCD nur in $58 \%$ der Fälle, jedoch nach $6-12,12-24$ bzw. $24-36 \mathrm{~h}$ in 77, 83 bzw. 100\% nachgewiesen [20]. Nach offenen Schädel-Hirn-Verletzungen und nach Dekompressions-Kraniotomien kann eine regional begrenzte zerebrale Zirkulation, z.B. infolge spontaner extra-intrakranieller Anastomosen, auftreten, sodass der zerebrale Zirkulationsstillstand durch die Doppler-/Duplexsonografie der Hirnbasisarterien nicht diagnostiziert werden kann und durch andere Perfusionsuntersuchungen nachzuweisen ist [1]. Nach großen offenen Schädel-Hirn-Verletzungen und vereinzelt bei sekundären Hirnschädigungen kommt es, wenn der intrakranielle Druck nicht über den mittleren arteriellen Druck ansteigen kann, nicht zu einem zerebralen Zirkulationsstillstand [1]. Diese Situation kann auch ohne Schädelverletzung bei Neugeborenen und bei Kindern bis zum vollendeten 2. Lebensjahr vorliegen, da sich die Schädelnähte und die Fontanellen erst im 2. Lebensjahr vollständig verschließen $[1,9]$. In diesen Fällen ist die Irreversibilität des Hirnfunktionsausfalls unter der Berücksichtigung des Alters und der Art der Hirnschädigung durch klinische Verlaufsuntersuchungen nach den normierten Wartezeiten oder durch geeignete elektrophysiologische Befunde nachzuweisen $[1,10]$.

Eine weitere wesentliche Limitation der transkraniellen Doppler-/Duplexsonografie ist die Abhängigkeit der Signalqualität von der Größe und Lage des transtemporalen Knochenfensters („Schallfensters“), die insbesondere bei älteren Patienten die Untersuchung intrakranieller Arterien einschränken oder unmöglich machen kann $[21,22]$. Für diesen Fall (und nur für diesen Fall) sieht die RL4-BÄK allerdings die Möglichkeit der Untersuchung der beidseitigen extrakraniellen Arterien (A. carotis interna und $A$. vertebralis) als ausreichend vor [1].

\section{Besonderheiten der Doppler-|Duplexsonografie $\nabla$}

Im Gegensatz zur Dopplersonografie ermöglicht die transkranielle Duplexsonografie durch die Darstellung des Hirngewebes, das die intrakraniellen Arterien umgibt, den Nachweis der Penetration des Ultraschalls durch den Schädelknochen und die Lokalisation der intrakraniellen Arterien jeweils unabhängig vom Vorliegen eines Blutflusses in diesen Arterien [1]. Als Signalver- 


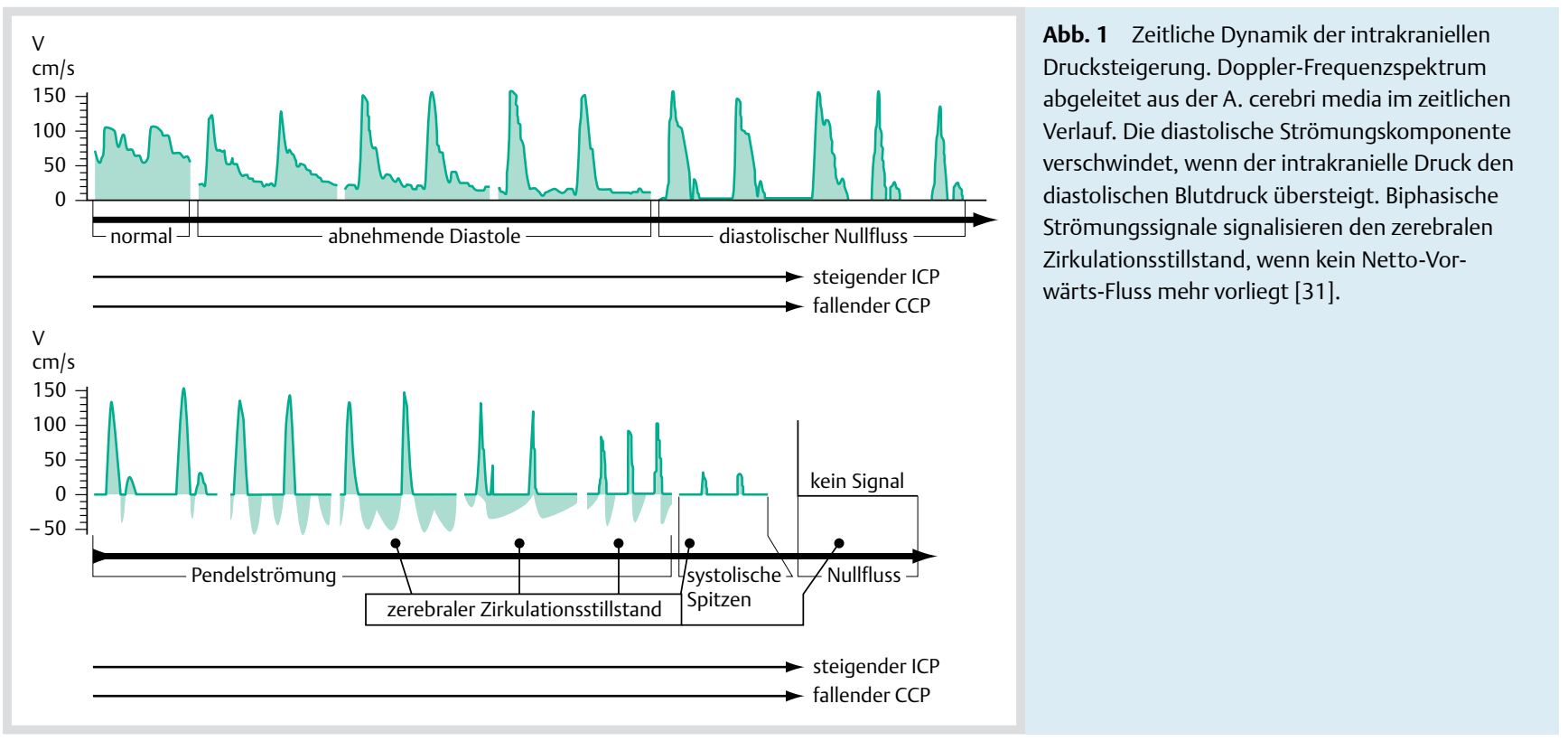

Tab. 2 Untersuchungsprogramm zum Nachweis des zerebralen Zirkulationsstillstandes.

\begin{tabular}{|c|c|c|c|}
\hline & \multirow{2}{*}{$\begin{array}{l}\text { BÄK Richtlinie } 1998 \\
\text { Dopplersonografie }\end{array}$} & \multicolumn{2}{|c|}{ BÄK Richtlinie 2016} \\
\hline & & $\begin{array}{l}\text { Doppler- } \\
\text { sonografie }\end{array}$ & $\begin{array}{l}\text { Duplex- } \\
\text { sonografie }\end{array}$ \\
\hline \multicolumn{4}{|l|}{ Extrakraniell bds. } \\
\hline $\mathrm{ACl}$ & + & $+\S$ & \\
\hline AV & + & $+\S$ & \\
\hline \multicolumn{4}{|l|}{ Intrakraniell bds. } \\
\hline $\mathrm{ACl}$ & + & + & + \\
\hline ACM & + & + & + \\
\hline $\mathrm{AV}\left(\mathrm{V}_{4}\right)$ & + & & + \\
\hline$A B$ & & & + \\
\hline „jede weitere“ & $+\$$ & $+\$$ & $+s$ \\
\hline \multicolumn{4}{|c|}{$\begin{array}{l}\text { § die Untersuchung der ACl und der AV sowohl in den extra- als auch in den intrakra } \\
\text { niellen Abschnitten wird nicht gefordert, da prinzipiell keine Notwendigkeit besteht, } \\
\text { eine Arterie an mehreren Stellen abzuleiten. \$ optional }\end{array}$} \\
\hline
\end{tabular}

stärker eingesetzte Ultraschallkontrastmittel erhöhen die Sensitivität der Duplexsonografie, um die für einen zerebralen Zirkulationsstillstand charakteristischen Strömungssignale an den intrakraniellen Arterien nachzuweisen [1,23, 24]. Die darzustellenden Gefäßabschnitte der A. carotis interna und der A. vertebralis sind in einigen Details unterschiedlich für die Dopplersonografie und die Duplexsonografie in der RL4-BÄK festgelegt worden [1], folgen aber dem gleichen Grundprinzip, dass jedes Gefäß nur noch an einem Abschnitt dargestellt werden muss ( $\bullet$ Tab. 2, $\odot$ Abb. 2). Dies wird in der RL4-BÄK ausführlich wie folgt begründet [1]: Die Untersuchung der Aa. carotides internae und der Aa. vertebrales in den extra- und intrakraniellen Abschnitten wird nicht gefordert, da prinzipiell keine Notwendigkeit besteht, eine Arterie an mehreren Stellen abzuleiten. Voraussetzung dafür ist allerdings, dass jede einzelne Ableitung technisch suffizient möglich ist und deren Untersuchungsergebnis für sich alleine eine eindeutige Schlussfolgerung erlaubt. Dies ist bei Ableitung der Aa. carotides internae intrakraniell und extrakraniell jeweils der Fall. Konsequenterweise kann, wenn die Ableitung an einer Stelle technisch nicht möglich ist, die Aussage durch Ableitung an einer anderen Stelle getroffen werden. Praktisch könnte die Forderung, trotz intrakranieller Signale des zerebralen Kreislaufstillstandes in den Aa. carotides internae, diese auch extrakraniell abzuleiten, bei technischen Hindernissen (z. B. zentraler Venenkatheter) sogar unnötigerweise die Diagnose doppler-/duplexsonografisch nicht erlauben. Bei duplexsonografischem Nachweis der für einen zerebralen Kreislaufstillstand charakteristischen Signale in der A. basilaris und beiden V4-Segmenten, von denen die Aa. cerebelli inferiores posteriores (sog. PICA) abgehen, besteht aus oben genannten Gründen ebenfalls keine Notwendigkeit, diese Signale auch in den extrakraniellen Aa. vertebrales abzuleiten. Die Forderung, diese Signale auch extrakraniell abzuleiten, könnte, aufgrund der Kollateralverbindungen der Aa. vertebrales zur jeweiligen A. carotis externa, extrakraniell unter Umständen zu Strömungssignalen führen, die unnötigerweise die Diagnose des zerebralen Kreislaufstillstandes duplexsonografisch nicht erlauben würden [1].

\section{Befunde des zerebralen Zirkulationsstillstandes $\nabla$}

Frühsystolische Spitzen, die eine systolische Maximalgeschwindigkeit von kleiner als $50 \mathrm{~cm} / \mathrm{s}$ und eine Dauer von unter $200 \mathrm{~ms}$ aufweisen, und biphasische Strömungssignale (oszillierende Strömungssignale, „Pendelfluss“) sind dopplersonografische Zeichen eines zerebralen Zirkulationsstillstandes ( $\bullet$ Abb. 3, 4).

Biphasische Strömungssignale sind nur dann verwertbar für einen zerebralen Zirkulationsstillstand, wenn das Integral (Fläche) der anterograden und retrograden Anteile des Doppler-Frequenzzeitspektrums innerhalb eines Herzzyklus gleich groß ist; es wird aber nicht gefordert, dass hier eine Messung durch das Ultraschallgerät erfolgen soll, in der Praxis wird das durch den Untersucher abgeschätzt [1]. Die biphasischen Strömungssignale nach zerebralem Zirkulationsstillstand können dabei durchaus noch relativ hohe Amplituden der orthograden systolischen Flusskomponente bis über $100 \mathrm{~cm} / \mathrm{s}$ haben, sind aber charakterisiert durch eine schmale monophasische 


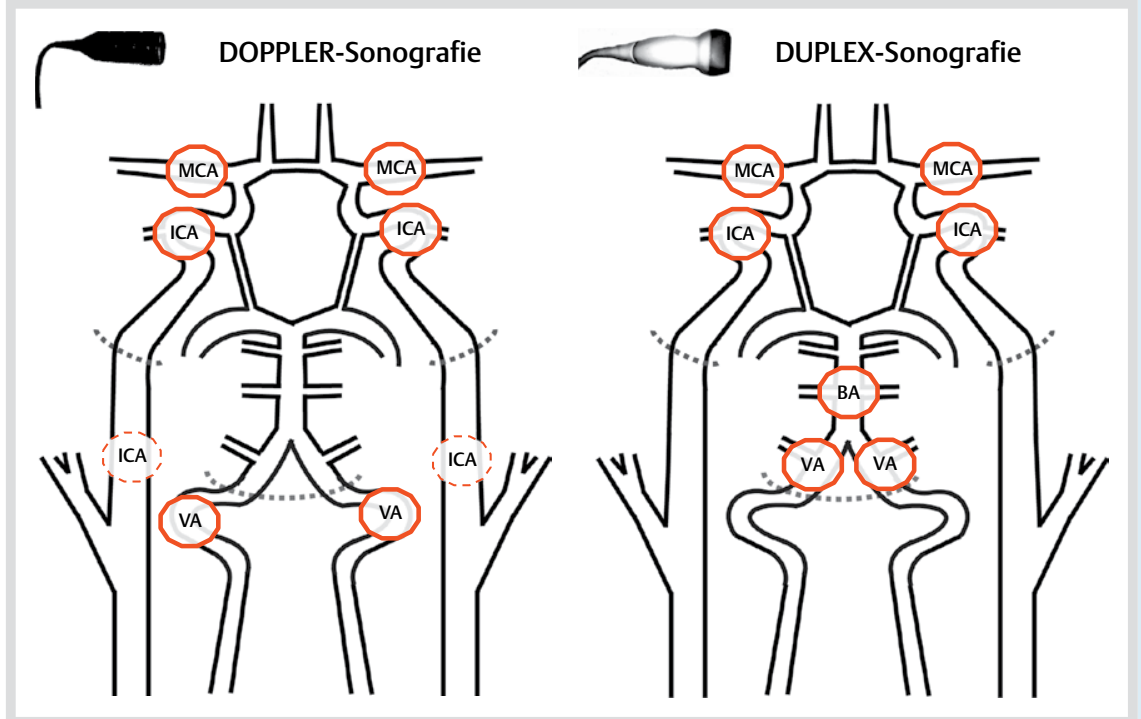

Abb. 2 Darzustellende Gefäße mit der Dopplerund Duplexsonografie zur Diagnostik des zerebralen Zirkulationsstillstandes.
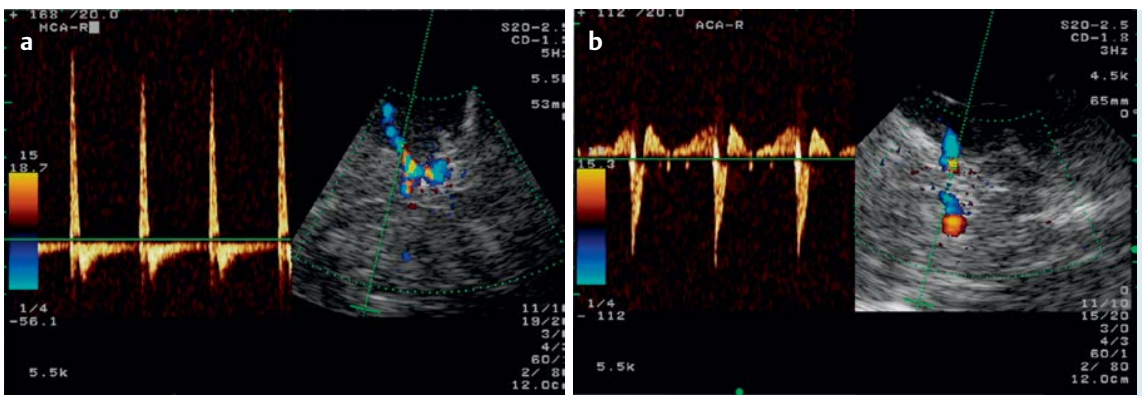

Abb. 3 Duplexsonografische Befunde beim zerebralen Zirkulationsstillstand. Die Bilder a und b zeigen typische biphasische Strömungssignale in $\operatorname{der} \mathrm{A}$. cerebri media und A. cerebri anterior. Die Bilder c und d zeigen „systolische Spitzen“ in der A. cerebri media und A. cerebri anterior.
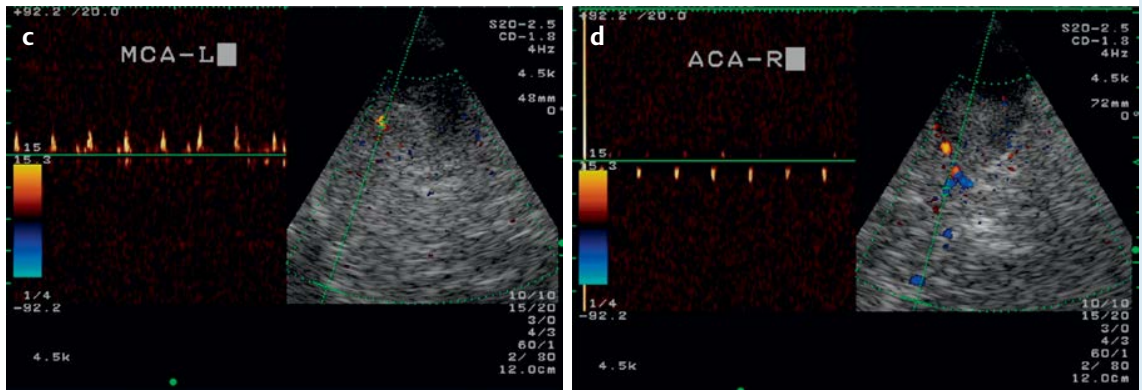

Konfiguration der orthograden systolischen Flusskomponente („systolische Spitzen“) [25,26]. Strömungssignale mit breiter oder angedeuteter biphasischer Konfiguration der orthograden systolischen Flusskomponente ( $\bullet$ Abb. 5) sollten nicht im Sinne eines zerebralen Zirkulationsstillstandes gewertet werden. Gelegentlich werden auch spontane oder posttraumatische arteriovenöse Shuntflüsse (AV-Fistel) detektiert, die die sonografische Diagnosestellung eines zerebralen Zirkulationsstillstandes ebenfalls nicht erlauben ( $\bullet$ Abb. 5).

Die RL4-BÄK gibt die folgenden Kriterien für die Feststellung des zerebralen Zirkulationsstillstandes mittels Doppler- oder Duplexsonografie vor [1]:

Zum Nachweis des zerebralen Zirkulationsstillstandes müssen im Abstand von mindestens 30 min

- mittels Dopplersonografie intrakraniell die Aa. cerebri mediae, Aa. carotides internae und eventuell detektierbare weitere Hirnbasisarterien, extrakraniell die Aa. vertebrales und, wenn die korrespondierenden intrakraniellen Gefäßsegmente nicht darstellbar sind, die Aa. carotides internae, oder
- mittels Duplexsonografie intrakraniell die M1-Segmente der Aa. cerebri mediae, die Aa. carotides internae, die V4-Segmente der Aa. vertebrales und die A. basilaris sowie eventuell detektierbare weitere Hirnbasisarterien

untersucht werden und dabei folgende Befunde nachweisbar sein:

- biphasische Strömungssignale (oszillierende Strömungssignale) mit gleich ausgeprägtem Integral der antero- und retrograden Komponente

oder

- frühsystolische Spitzen, die kleiner als $50 \mathrm{~cm} / \mathrm{s}$ sind und unter $200 \mathrm{~ms}$ anhalten, wobei kein weiteres Strömungssignal im verbleibenden Herzzyklus detektierbar sein darf.

Ein Fehlen der Strömungssignale bei transkranieller Beschallung der Hirnbasisarterien kann nur dann als sicheres Zeichen eines zerebralen Zirkulationsstillstandes gewertet werden, wenn derselbe Untersucher mit gleicher Geräteeinstellung während einer früheren Untersuchung eindeutig ableitbare intrakranielle Strömungssignale dokumentiert hat, oder wenn an den extrakraniellen hirnversorgenden Arterien (Aa. carotides internae, Aa. vertebrales) die Zeichen des zerebralen Zirkulationsstillstandes 


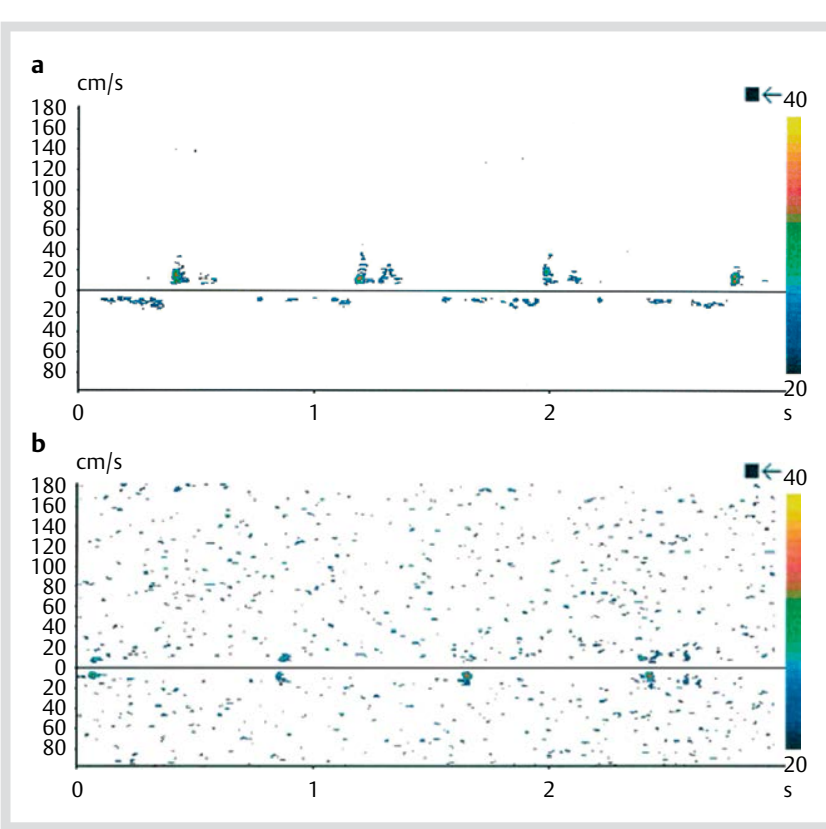

Abb. 4 Dopplersonografische Befunde beim zerebralen Zirkulationsstillstand. Das in Bild a dargestellte Doppler-Frequenzspektrum zeigt ein biphasisches Strömungssignal in der A. cerebri media. Das in Bild b dargestellte Dopplerfrequenzspektrum zeigt „systolische Spitzen“ in der A. cerebri media. Aufgrund der begrenzten Qualität des Doppler-Frequenzspektrums und der niedrigen Amplitude des Flusssignals muss mit hoher Verstärkung (Sensitivität) und niedrigem Wandfilter untersucht werden.
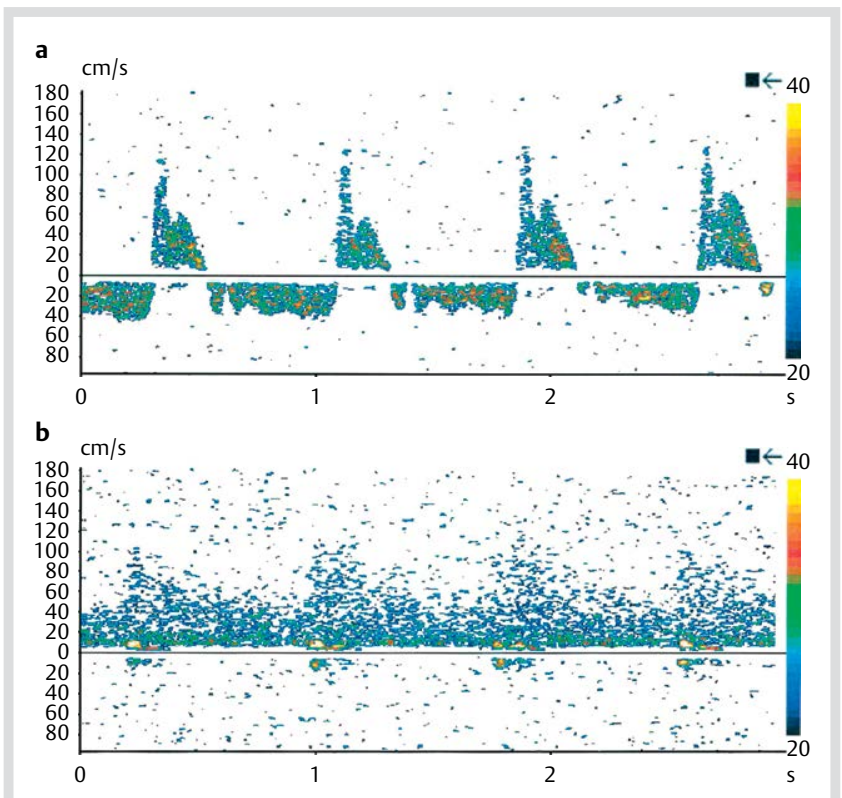

Abb. 5 Dopplersonografische Befunde, die mit der Diagnose des zerebralen Zirkulationsstillstandes nicht vereinbar sind. Das in Bild a dargestellte Doppler-Frequenzspektrum zeigt einen großvolumigen Pendelfluss mit breiter, biphasischer Konfiguration der orthograden systolischen Flusskomponente; hier kann zwar ein zerebraler Zirkulationsstillstand vorliegen, jedoch ist der zerebrale Zirkulationsstillstand mit diesem Dopplerbefund nicht zu diagnostizieren, da eine Restperfusion von Hirnarealen hiermit nicht sicher ausgeschlossen werden kann. Das in Bild b dargestellte Doppler-Frequenzspektrum zeigt einen arterio-venösen Shuntfluss, überlagert durch systolische Spitzen (proximale A. cerebri media). Auch dieser Befund erlaubt die Ultraschalldiagnose eines zerebralen Zirkulationsstillstandes nicht. In beiden Fällen a, b konnte der zerebrale Zirkulationsstillstand schließlich mittels Perfusions-Szintigrafie bewiesen werden. nachweisbar sind [1]. Primär fehlende Strömungssignale dürfen nicht als Irreversibilitätsnachweis gewertet werden, da intrakranielle Strömungssignale bei unzureichender Schalltransmission durch den Knochen, intrakranieller Luftansammlung und massiver Gefäßverlagerung trotz noch erhaltener Hirndurchblutung fehlen können [10]. Die Befunderhebung mittels Doppler- und Duplexsonografie kann durch eine Reihe von Fehlermöglichkeiten beeinträchtigt werden ( $\bullet$ Tab. 3 ), welche unbedingt beachtet oder ausgeschlossen werden müssen [27,28]. Die zweite Untersuchung in zeitlichem Abstand von $30 \mathrm{~min}$ soll sicherstellen, dass es sich nicht nur um einen kurzzeitigen, reversiblen Zirkulationsstillstand handelt. Dabei ist der Zeitpunkt des Beginns der jeweiligen sonografischen Untersuchung maßgeblich; das heißt, der Beginn der zweiten Untersuchung kann frühestens $30 \mathrm{~min}$ nach Beginn der Erstuntersuchung (oder aber auch später) begonnen werden [29]. Im Untersuchungsbefund wie auch in den Protokollbögen muss dementsprechend diese Zeitspanne $>30$ min dokumentiert sein.

Wird die Doppler- oder Duplexsonografie gemäß den hier ausführlich dargestellten Vorgaben und zeitlich nach der standardisierten Feststellung der klinischen Symptome des Hirnfunktionsausfalls gemäß RL4-BÄK durchgeführt und der zerebrale Zirkulationsstillstand bei zweimaliger Untersuchung im Abstand von 30 min bestätigt, sind nach Stand der wissenschaftlichen Erkenntnisse keine falsch-positiven Ergebnisse zu erwarten (somit eine 100\%ige Spezifität der Doppler-/Duplexsonografie) $[13,28-29]$

\section{Apparative Anforderungen \\ $\nabla$}

Grundsätzlich können alle kommerziell in Deutschland verfügbaren Ultraschall-Systeme, die für die Untersuchung der extraund intrakraniellen hirnversorgenden Arterien konfiguriert sind, verwendet werden. Voraussetzung sind geeignete Sonden (Dopplersonografie [extrakraniell: 4MHz-Sonde, transkraniell: $2 \mathrm{MHz}-S o n d e$, Duplexsonografie [extrakraniell: 5-10 MHz-Sonde, transkraniell: 1-2,5 MHz-Sonde]). Um die Detektion niedrigvolumiger oder langsamer Flüsse mittels der Doppler- oder Duplexsonografie sicher zu stellen, sind geeignete Geräteeinstellungen zu wählen ( $\bullet$ Tab. 4).

\section{Anforderungen an den Untersucher \\ $\nabla$}

Die Untersuchung muss durch einen in dieser Methode speziell erfahrenen Arzt durchgeführt werden [1]. Eine medizinisch-technische Assistentin kann also diese Untersuchung im Rahmen der Hirntoddiagnostik nicht durchführen, wohl aber ein qualifizierter Arzt, der diese Leistung konsiliarisch erbringt. In den Fachgremien der DEGUM und DGKN hat es eine intensive Diskussion darüber gegeben, ob ein Zertifikat des Untersuchers als Voraussetzung für die Durchführung der sonografischen Diagnostik im Rahmen der Hirntoddiagnostik zu fordern ist. Davon ist schließlich abgesehen worden, um langjährig erfahrenen Untersuchern in dieser Indikation, die evtl. kein Zertifikat haben, weiterhin die Möglichkeit der Durchführung zu geben. Dennoch sehen die Autoren es als wünschenswert und sinnvoll an, dass die in dieser Indikation tätigen Ärzte ein Zertifikat der DEGUM oder DGKN für die Doppler-/Duplexsonografie der hirnversorgenden Arterien erwerben, um damit ihre Kompetenz formal zu dokumentieren. 
Tab. 3 Fallstricke (modifiziert nach [28]).

1. Primär fehlende Strömungssignale dürfen für sich alleine nicht als Irreversibilitätsnachweis gewertet werden.

2. Falsch-negative Befunde können bei größeren Knochendefekten oder Kleinkindern mit noch nicht verschlossener Fontanelle erhoben werden.

3. Starke Tachykardie (Tachyarrhythmie, Sinustachykardie>120/min) kann durch Überlagerung der Pulswellen eine noch vorhandene Strömung vortäuschen.

4. Starke Bradykardie kann die Dauer systolischer Spitzen auf über $200 \mathrm{~ms}$ verlängern, was die Wertung im Sinne eines zerebralen Zirkulationsstillstandes gemäß RL4-BÄK ausschließt.

5. Gelegentlich intrakraniell nachweisbare, schwach pulsatile venöse Flusssignale können zu falsch-negativen Befunden führen (vermeintlicher arterieller zerebraler Blutfluss).

6. Artefakte oder Flussveränderungen aufgrund von intraaortaler Ballonpumpe (IABP), arteriovenöser extrakorporaler Membranoxygenierung (ECMO) oder Beatmung können zu schwer interpretierbaren Befunden führen.

7. Duplexsonografisch kann selbst bei Vorliegen typischer Flusssignale (systolische Spitzen, biphasische Strömungssignale) beidseits in der A. cerebri media, A. cerebri anterior, A. cerebri posterior, A. basilaris und A. vertebralis (V4-Segment) die Diagnose des Zirkulationsstillstandes gemäß RL4-BÄK nicht gestellt werden, wenn der Fluss in der intrakraniellen A. carotis interna in Richtung A. ophthalmica erhalten ist. Diese falsch-negative Befundkonstellation wurde in der RL4-BÄK zur Vermeidung von irrtümlichen Gefäßzuordnungen bewusst in Kauf genommen. In einem solchen Fall kann die CTA als alternatives Verfahren erwogen werden, da dort die Diagnose eines zerebralen Zirkulationsstandes trotz eines residualen Flusses in der die A. ophthalmica versorgenden A. carotis interna zulässig ist

Tab. 4 Untersuchungstechnik zum Nachweis des Zirkulationsstillstandes.

\section{Transkranielle Dopplersonografie}

1. Den arteriellen Blutdruck messen: arterieller Mitteldruck bei Erwachsenen $>60 \mathrm{~mm} \mathrm{Hg}$.

2. Mit hoher Sende- und Empfangsleistung beginnen, da sonst Signale mit schwacher Intensität nicht erfasst werden können.

3. Die Hüllkurve ausschalten, da sie mehr verwirrt als nützt.

4. Den Wandfilter unter $50 \mathrm{~Hz}$ senken, da sonst z. B. systolische Spitzen ausgefiltert werden.

5. Das Messvolumen möglichst groß wählen ( $15 \mathrm{~mm})$.

6. Die Untersuchung in größerer Tiefe beginnen, da distale Äste der A. cerebri media u. U. komprimiert und verlagert sein können.

\section{Transkranielle (Farb-)Duplexsonografie *}

1. Den arteriellen Blutdruck messen: arterieller Mitteldruck bei Erwachsenen $>60 \mathrm{~mm} \mathrm{Hg}$.

2. Farbfenster nicht zu groß einstellen.

3. Pulsrepetitionsfrequenz (PRF) für niedrige Flusssignale optimieren (reduzieren).

4. Farbgain (Empfangsleistung für den Farbmodus) erhöhen.

5. Korrekte Schallebenen auswählen, dabei anatomische B-Bild Landmarken zum Aufsuchen der Gefäße nutzen: a) obere Ponsebene (Treffpunkt von Keilbeinflügel und Felsenbein) für ICA-Siphon und A. ophthalmica, b) untere Ponsebene (Felsenbein) für petrosale ICA, c) Mittelhirnebene für Darstellung der Circulus-Willisii-Ebene.

6. Bei fehlendem intrakraniellen Flusssignal, jedoch noch erkennbaren Strukturen im B-Bild Gabe eines Echosignalverstärkers erwägen.

* Die Bildgebung erlaubt eine exakte topografische Zuordnung der Dopplersignale, die man für die Diagnose des Zirkulationsstillstandes verwertet

\section{Anforderungen an den Befundbericht}

Gemäß den aktualisierten Empfehlungen der DGKN soll der Befundbericht neben den üblichen Informationen (Fragestellung, Befundbeschreibung, Beurteilung) die folgenden Angaben enthalten [10]: Untersuchungszeit (Datum, Uhrzeit), Blutdruck (arterieller Mitteldruck oder systolischer und diastolischer Blutdruck), Benennung der dargestellten Gefäße mit der Art der jeweils detektierten Strömungssignale (frühsystolische Spitzen, biphasische Strömung mit gleich ausgeprägtem Integral der antero- und retrograden Komponente, erhaltene Perfusion, kein Strömungssignal nachweisbar). Im Falle fehlender transkranieller Strömungssignale ggf. Vergleich mit der von demselben Untersucher durchgeführten Voruntersuchung beschreiben, Name des untersuchenden/befundenden Arztes, und Form der Archivierung der abgeleiteten Strömungssignale angeben (Papierausdruck oder digital). Wenn im Anschluss an die Feststellung des irreversiblen Hirnfunktionsausfalls (ggf. mit Durchführung der Doppler- bzw. Duplexsonografie) eine Organ- oder Gewebespende durchgeführt wird, erfolgt die Archivierung für 30 (!) Jahre [1].

\section{Vorgehen bei unvollständiger Untersuchbarkeit oder unsicheren Befunden}

Wenn die doppler-/duplexsonografische Untersuchung nicht vollständig gemäß den Vorgaben der RL4-BÄK abgeschlossen werden kann, ist der Einsatz einer alternativen Methode zum Nachweis des zerebralen Zirkulationsstillstandes (KM-gestützte CT-Angiografie, Perfusions-Szintigrafie) möglich [1]. Dies gilt ebenso in Fällen, in denen extra-intrakranielle Shuntflüsse in der Doppler-/Duplexsonografie nachgewiesen werden. Diese Konstellation kann nach sekundären Hirnschädigungen beobachtet werden, wenn kein schweres Hirnödem, kein maximal erhöhter Hirndruck und damit kein zerebraler Zirkulationsstillstand entsteht. Des Weiteren besteht die - in der Praxis seltene - Möglichkeit, dass mit dem zunehmenden Entstehen von Hirnnekrosen die eingetretenen dopplersonografischen Zeichen des Zirkulationsstillstandes (systolische Spitzen) partiell wieder verschwinden und dass wieder schwache orthograde Flüsse auftreten [30]. Gemäß den Vorgaben der RL4-BÄK ist neben dem Einsatz einer anderen Methode zum Nachweis des zerebralen Zirkulationsstillstandes alternativ unter Beachtung der jeweilig erforderlichen Voraussetzungen die Möglichkeit des Einsatzes einer elektrophysiologischen Methode oder der 2. klinischen Untersuchung nach entsprechender Wartezeit gegeben $[1,10]$. 
Interessenkonflikt: Die Autoren geben an, dass kein Interessenkonflikt besteht.

\section{Literatur}

1 Wissenschaftlicher Beirat der Bundesärztekammer. Richtlinie gemäß § 16 Abs. 1S. 1 Nr. 1 TPG für die Regeln zur Feststellung des Todes nach $\S 3$ Abs. 1S. 1 Nr. 2 TPG und die Verfahrensregeln zur Feststellung des endgültigen, nicht behebbaren Ausfalls der Gesamtfunktion des Großhirns, des Kleinhirns und des Hirnstamms nach $\S 3$ Abs. 2 Nr. 2 TPG, Vierte Fortschreibung. Dtsch Ärztebl 2015; 112: A-1256

2 Wahlster S, Wijdicks EF, Patel PV et al. Brain death declaration: Practices and perceptions worldwide. Neurology 2015; 84: 1870-1879

3 Vicenzini E, Pro S, Pulitano $P$ et al. Current practice of brain death determination and use of confirmatory tests in an Italian University hospital: a report of 66 cases. Minerva Anestesiol 2013; 79: 485-491

4 Hoffmann 0 , Masuhr F. Zugang zur Hirntoddiagnostik. Nervenarzt 2014; 85: 1573-1581

5 Orban JC, Ferret E, Jambou $P$ et al. Confirmation of brain death diagnosis: A study on French practice. Anaesth Crit Care Pain Med 2015; 34: $145-150$

6 Escudero D, Valentín MO, Escalante JL et al. Intensive care practices in brain death diagnosis and organ donation. Anaesthesia 2015; 70: 1130-1139

7 Consensus Group on Transcranial Doppler in Diagnosis of Brain Death. Latin American consensus on the use of transcranial Doppler in the diagnosis of brain death. Rev Bras Ter Intensiva 2014; 26: 240-252

8 Alexandrov AV, Sloan MA, Tegeler $\mathrm{CH}$ et al. Practice standards for transcranial Doppler (TCD) ultrasound. Part II. Clinical indications and expected outcomes. J Neuroimaging 2012; 22: 215-224

9 Ducrocq X, Hassler W, Moritake Ket al. Consensus opinion on diagnosis of cerebral circulatory arrest using Doppler-sonography: Task Force Group on cerebral death of the Neurosonology Research Group of the World Federation of Neurology. J Neurol Sci 1998; 159: 145-150

10 Walter U, Brandt SA, Ferbert A et al. Empfehlungen der Deutschen Gesellschaft für Klinische Neurophysiologie und Funktionelle Bildgebung zur Diagnostik des irreversiblen Hirnfunktionsausfalls. Klin Neurophysiol 2016; 47: 16-20

11 Welschehold S, Boor S, Reuland K et al. Technical aids in the diagnosis of brain death: a comparison of SEP, AEP, EEG, TCD and CT angiography. Dtsch Arztebl Int 2012; 109: 624-630

12 von Reutern GM. Zerebraler Zirkulationsstillstand. Diagnostik mit der Dopplersonographie. Dtsch Ärztebl 1991; 88: A-4379-4385

13 Ducrocq X, Braun M, Debouverie $M$ et al. Brain death and transcranial Doppler: experience in 130 cases of brain dead patients. J Neurol Sci 1998; 160: 41-46

14 Hadani M, Bruk B, Ram Z et al. Application of transcranial Doppler ultrasonography for the diagnosis of brain death. Intens Care Med 1999; 25: 822-828
15 de Freitas GR, André C. Sensitivity of transcranial Doppler for confirming brain death: a prospective study of 270 cases. Acta Neurol Scand 2006; 113: 426-432

16 Marinoni M, Alari F, Mastronardi $V$ et al. The relevance of early TCD monitoring in the intensive care units for the confirming of brain death diagnosis. Neurol Sci 2011; 32: 73-77

17 Poularas J, Karakitsos D, Kouraklis $G$ et al. Comparison between transcranial color Doppler ultrasonography and angiography in the confirmation of brain death. Transplant Proc 2006; 38: 1213-1217

18 Haque IU, Zaritsky AL. Analysis of the evidence for the lower limit of systolic and mean arterial pressure in children. Pediatr Crit Care Med 2007; 8: 138-144

19 Jackson LV, Thalange NK, Cole TJ. Blood pressure centiles for Great Britain. Arch Dis Child 2007; 92: 298-303

20 Kuo JR, Chen CF, Chio CC et al. Time dependent validity in the diagnosis of brain death using transcranial Doppler sonography. J Neurol Neurosurg Psychiatry 2006; 77: 646-649

21 Nedelmann M, Stolz E, Gerriets $T$ et al. Consensus recommendations for transcranial color-coded duplex sonography for the assessment of intracranial arteries in clinical trials on acute stroke. Stroke 2009; 40: 3238-3244

22 Krejza J, Swiat M, Pawlak MA et al. Suitability of temporal bone acoustic window: conventional TCD versus transcranial color-coded duplex sonography. J Neuroimaging 2007; 17: 311-314

23 Llompart-Pou JA, Abadal JM, Velasco J et al. Contrast-enhanced transcranial color sonography in the diagnosis of cerebral circulatory arrest. Transplant Proc 2009; 41: 1466-1468

24 Welschehold S, Geisel F, Beyer C et al. Contrast-enhanced transcranial Doppler ultrasonography in the diagnosis of brain death. J Neurol Neurosurg Psychiatry 2013; 84: 939-940

25 Hassler W, Steinmetz H, Pirschel J. Transcranial Doppler study of intracranial circulatory arrest. J Neurosurg 1989; 71: 195-201

26 Poularas J, Karakitsos D, Kouraklis G et al. Comparison between transcranial color Doppler ultrasonography and angiography in the confirmation of brain death. Transplant Proc 2006; 38: 1213-1217

27 Harrer JU, Eyding J, Ritter $M$ et al. Neurosonografie in der neurologischen Notfall- und Intensivmedizin: Monitoring des erhöhten intrakraniellen Druckes, Hirntoddiagnostik und Untersuchung der zerebralen Autoregulation - Teil 2. Ultraschall Med 2012; 33: 320-331

28 Günther A, Llompart-Pou JA, Klingner C et al. Sonografische Methoden in der Hirntoddiagnostik. Klin Neurophysiol 2014; 45: 168-175

29 Brandt SA, Walter U, Schreiber S. Anwendung der aktuellen Richtlinie zur Feststellung des irreversiblen Hirnfunktionsausfalls. Intensivmedizin up2date 2016; 12: 17-40

30 Burger R, Schlake HP, Seybold S et al. Die Wertigkeit der transkraniellen Dopplersonographie im Vergleich zu szintigraphischen Verfahren und EEG in der Hirntoddiagnostik. Zentralbl Neurochir 2000; 61: 7-13

31 Kaps M, von Reutern GM, Stolz E, von Büdingen HJ. Ultraschall in der Neurologie. 2. korrigierte Auflage. Stuttgart: Georg Thieme Verlag; 2005 